\section{Venomics and antivenomics data: Current and future perspective}

\section{Soodeh Omidi ${ }^{1}$, Masoumeh Mehrpouya ${ }^{2}$, Morteza Oladnabi ${ }^{3-5 *}$, Abbas Azadmehr ${ }^{6}$, Fatemeh Kazemi-Lomedasht ${ }^{7}$ and Najmeh Yardehnavi ${ }^{8 *}$}

\author{
${ }^{1}$ Department of Medical Genetics, School of Advanced Technologies in Medicine, Golestan \\ University of Medical Sciences, Gorgan, Iran \\ 2Department of Medical Biotechnology, School of Advanced Technologies in Medicine, Golestan \\ University of Medical Sciences, Gorgan, Iran \\ ${ }^{3}$ Stem Cell Research Center, Golestan University of Medical Sciences, Gorgan, Iran \\ ${ }^{4}$ Ischemic Disorders Research Center, Golestan University of Medical Sciences, Gorgan, Iran \\ ${ }^{5}$ Gorgan Congenital Malformations Research Center, Golestan University of Medical Sciences, \\ Gorgan, Iran \\ ${ }^{6}$ Department of Medical Immunology, Babol University of Medical Sciences, Babol, Iran \\ ${ }^{7}$ Venom and Biotherapeutics Molecules Laboratory, Biotechnology Department, Biotechnology \\ Research Center, Pasteur Institute of Iran, Tehran, Iran \\ ${ }^{8}$ Department of Medical Biotechnology, School of Medicine, Mashhad University of Medical \\ Sciences, Mashhad, Iran
}

\section{Abstract}

Venom has a very complex and exclusive nature which has been introduced by recent advances in omics technologists. These methods have revealed a new insight into venom studies as venomics. Envenoming by venomous animals is a global concern due to the distribution of important medical species around the world. Treatment of envenomed victims is dependent on accurate and fast identification of animal species with different detection methods. In recent years, new methods have been introduced based on molecular and immunological techniques. Precise diagnosis of species of venomous animals is an essential factor for treatment with specific antivenoms. Venomics and antivenomics data sets help in the selection of specific antivenoms or production of novel antivenoms with greater efficacies.

\section{More Information}

*Address for Correspondence:

Morteza Oladnabi, PhD, Department of Medical Genetics, Faculty of Advanced Medical Technologies, Golestan University of Medical Sciences, Gorgan, Iran, Tel: +98 1732430565; Fax: +98 1732430565;

Email: oladnabidozin@yahoo.com

Najmeh Yardehnavi, MD, PhD, Department of Medical Biotechnology, School of Medicine, Mashhad University of Medical Sciences, Mashhad, Iran,

Email: yardehnavi.najmeh@gmail.com

Submitted: March 07, 2021

Approved: April 19, 2021

Published: April 20, 2021

How to cite this article: Omidi S, Mehrpouya M, Oladnabi M, Azadmehr A, Kazemi-Lomedasht F, et al. Venomics and antivenomics data: Current and future perspective. Arch Biotechnol Biomed. 2021; 5: 026-031.

DOI: 10.29328/journal.abb.1001025

Copyright: @ 2021 Omidi S, et al. This is an open access article distributed under the Creative Commons Attribution License, which permits unrestricted use, distribution, and reproduction in any medium, provided the original work is properly cited.

Keywords: Venomics; Antivenomics; Venom; Antivenom

D) Check for updates

(9) OPEN ACCESS

\section{Introduction}

In the course of evolution, multi-gene families have arisen from sequential duplication of genes that are involved in the production of physiological proteins. Then duplicated copies of old genes in the venom glands can produce proteins with a new function as toxins [1]. The high rate of non-synonymous mutations in the multi-gene families and low repeat genes of toxins demonstrate the pressure of selection on the coding sequences which result in a diversity of gene sequences and venom functions $[2,3]$. Both of the evolutionary mechanisms including natural selection and genetic drift cooperate for venom evolution and for the success of venomous animals in the interactions with other animals over the years. Components with a high abundance are experiencing powerful forces of positive selection and strong drift forces. In contrast, weak forces of selective constraint lead to replacement of deleterious mutations in the gene sequences [4]. Despite the increasing venom efficacy for surviving through venom evolution, there are many non-venomous animals that have lost their venom system in the evolutionary process due to the optimization of costs and benefits [5].

Generally, physiological elements and venom are present in a cycle whose diversities are dependent on each other and their coevolution is the cause of extensive diversity of venom contents. Therefore, venoms have been evolved into a biological substance with more molecular diversity, activity, and specificity which can target those physiological elements. These results are evidence of a connection between humans and venom not only as a predator and prey interaction but also beyond this interaction [5]. Interestingly, the wellestablished complex nature of venoms, especially with the help of recent advances in venomics studies, draws the 
attention of researchers even more than before to the venom and venomous animals. In this review, we will discuss about role of venomics and antivenomics dataset in development of specific antivenoms for treatment.

\section{Venomics}

In recent years, the studies of animal venoms thanks to the advances in highly sensitive and high-throughput techniques such as genomics, transcriptomics, proteomics, and metabolomics have introduced large-scale data from venoms known as venomics, and opened up new opportunities for biological and clinical venom applications [6-12]. Before improvements of these technologies, venom studies mostly focused on a single toxin or component of venoms and investigated their structure and function. Advances in new methods such as reverse-phase high-performance liquid chromatography (RP-HPLC) and mass spectrometry (MS) help us identify various animal venoms and their content. Subsequently, the breadth of our information assists in phylogenetic studies and following the trail of the evolutionary process of the venoms as the animal venom is modulated by natural pressures such as genetic mutations, genetic drift, and natural selection for optimal adaptation [13]. In addition to natural pressures for venom diversity and venom adaptation for a specific function, the composition and abundance of venom components are influenced by local environmental conditions, geographical and genetic separation factors causing intraspecific diversity in animal venom peptides or proteins for adaptation to their environment [14,15]. Venom development is an important event which is the reason for survival of venomous animals for many years. Venomics data can provide information for phylogenic studies. The shared types of peptides or proteins provide information from recruitment patterns of animal venoms such that the common types of peptides or proteins may be recruited into the proteome before lineage-7splitting of considered species during the evolution $[16,17]$.

For instance, venom proteome information of about 132 snake species which had been investigated in the last ten years was collected for comparative data analysis of snake venom. In addition to four dominant and six secondary protein classes in these species, many differences were identified in the venom composition and abundance of components [13]. Snake venom with a complex mixture of several enzymes, proteins, polypeptides, and many other components is a rich source of potent biologically active molecules such as toxins with high specificity, efficacy, and fast performance in targeting; these are first beneficial for defending and hunting, while also conferring many medical benefits. There are approximately 375 venomous snake species worldwide and their venom is an excellent source of novel toxins. The Snake Venom DataBase (SVDB) contains all information on venomous snakes, their venom compositions, and function (https://www.snakevenomdb.org/).
In addition to snakes, many studies have focused on other dangerous and venomous animals such as cone snails, scorpions, and spiders. From the beginning of venomics analysis, different species of scorpions have been considered by researchers. The genome, transcriptome, and proteome analysis of different species of scorpions demonstrates the molecular diversity of their genes and proteins including typical and atypical protein families where a toxin family is a major group of proteins in their venom such as sodium and potassium channel toxins. Also, venomics analysis has introduced many novel toxins [18-22]. Interestingly, in addition to the proteome profile of venom, the presence of several chemical elements such as sodium, potassium, calcium, copper, zinc, and manganese with various concentrations in their venom assists in representing the way venoms function and their pharmacological applications [23]. Cone snails are other important venomous animals with about 800 species with each producing a unique proteome profile with over 1000 components including various cysteine-rich peptides or conopeptides. Venomics data of cone snails can contribute to the discovery of venom composition and characterization of its components as well as increasing the number of novel peptides. They also accelerate demystifying the cone snails venom complexity which can help in the application of toxins [24]. For example, differences in the quality and quantity of venom in various specimens from different sections of venom duct suggest that different sections of venom duct are specialized for production of specific conotoxins. as Also, the presence of post-translational modified peptides for enhanced diversity and the presence of alternative cleavage sites in the sequence of components [25], the regional variations between specimens from the same species in the same geographical region [26-28], the complexity depending on the breadth of dietary [29] as well as differences given the stimulation types including predatory or defensive venom are of interest $[30,31]$. Many novel components with pharmacological features have been introduced in spider's venom through ongoing venomics data. Venomics data indicate that many toxins in spider's venom are cysteine-rich peptides with 3-9 $\mathrm{kDa}$ molecular weight [32], and the Inhibitor Cysteine Knot (ICK) motif is the most structural motif of these toxins among many various species of spiders. These results confirm the conservation of these classes of toxins among them [33-35]. Interestingly, the spider venom changes during its lifetime and development; these changes can be a result of various predation-prey interactions in different periods of life such as demand of mate for males with these changes being examples of constant ontogenetic changes [36]. These are examples of venom diversity and today there is much evidence of venom variations from various animals by venomics studies.

Recently, venomics data are improving by applying new methods such as a rapid, efficient, and easy methods through on-chip technology [37] as well as new insights such as using proteomics technology for studying the composition 
of protein cargo of extracellular vesicles [38]. Evolution of our information from venomous animals and their venom in recent years with different approaches first provides a comprehensive catalog of venom composition and venom diversity for their clinical and pharmacological applications. It also helps us in the management of health care services for venomous victims as they need their special remedy according to each species of venomous animals. Interestingly, although the venom composition is similar between many species and most of the components belong to the main functional protein classes, they have various amino acid sequences and epitopes in their structures which may be the cause of the need for specific antiserums against each species [39].

One of the causes of morbidity and mortality worldwide is animal bites especially in Africa and South-East Asia. A large number of envenoming is associated with snake bites such that each year more than 5 million people are bitten, 2.4 million people are envenomed and 94,000-125,000 are dead by snakes (https://www.who.int/en/news-room/fact-sheets/ detail/animal-bites\#). Death or other health consequences of envenomed people highlight the need for providing timely health care services.

Envenoming is a serious cause of morbidity and mortality in various regions of the world especially in tropical and subtropical regions particularly snakebites which has been introduced as a neglected tropical disease by World Health Organization (WHO). A common complication of envenoming by various species of snakes is neurotoxicity. Acute neuromuscular paralysis is the major manifestation of neurotoxicity and is the cause of severe health consequences of snakebites [40]. The neurotoxicity by blockade of neurotransmission, as well as disruption of blood coagulation and triggering immune system type 2 are prevalent and wellknown consequences of envenomation of numerous species of animals as introduced by toxicology studies in humans [5]. Since envenomation by venomous animals is a human health concern, recent advances in the detection of venomous animal species based on antigen and antibody interaction [41] as well as molecular detection [42] for common species in the particular regions of the world facilitate the treatment of envenomed patients through specific antivenoms.

\section{Diagnosis of envenomation}

Diagnosis of envenomation is based on clinical and laboratory factors. In addition to general laboratory diagnosis by blood parameters, identification of venom-specific antigens or antibodies against these antigens by examining the blood or other body fluids for determining the animal species and effective antivenoms has played an important role in the treatment of envenomed victims. Therefore, for the treatment of envenomed victims, there are two important factors including accurate diagnosis of animal species and specific antivenom for the treatment. Methods based on immunological techniques have been used by researchers, such as gel immunodiffusion, immunofluorescence, hemagglutination, immunoelectrophoresis, radioimmunoassay (RIA), enzymelinked immunosorbent assay (ELISA/EIA), and optical immunoassay [43,41]. Among these bio-detection systems based on immunological reactions, RIA and ELISA are more common due to their sufficient specificity and sensitivity. The ELISA technique provides more benefits such as inexpensive, uncomplicated, stable reagents, different samples for detection including blood and other biological fluids [44]. From many years ago, microtiter plates coated with specific venom antibodies or venom antigens have been used in direct and indirect ELISA respectively [41] such that the detection based on ELISA has been established around the world even as commercial kits for common snakes in susceptible regions such as Australia [45,46], Vietnam [47], and Egypt [48]. Another rapid, specific and sensitive snake venom detection kit has been utilized based on optical immunoassay (OIA) for four important snakes of South Vietnam. In this method, the physical changes in the thickness of molecular thin films caused by the specific binding of antigen and antibody are visible through light reflectance from gold into purple or blue color which depends on the thickness of the optical layer or the quantity of antigen-antibody complex formation [49]. In recent years, many efforts have been made to enhance the accuracy, sensitivity, specificity, and to reduce the test response time, test costs, and portability based on the antigenantibody complex for timely detection of venomous animal and provision of health care services. These services include detection based on surface plasmon resonance spectroscopy or detection based on chip with a highly sensitive transducer and quartz crystal microbalance (QCM), which are integrated by biosensors for venom detection $[50,51]$.

The application of molecular techniques for identification of animal venoms was introduced by employing polymerase chain reaction (PCR) in 2001. Due to the lack of sufficient information about gene sequences, the cobrotoxin-encoding gene was selected which could distinguish monocellate cobra from other common snakes in Thailand [42]. Then, in 2005 DNA barcoding method was employed for snake species recognition from dried snake venoms. In this approach, DNA extraction and sequencing of a mitochondrial $12 \mathrm{~S}$ gene supported the identification of snake species via comparing sequences with the GenBank database and was helpful in building the DNA barcodes of that species which can be used for matching the barcode sequences from unknown specimens [52]. DNA barcodes of other mitochondrial genes such as COI and Cytb genes from 16 snake species were constructed in Thailand. The results showed that COI gene is better for DNA barcoding and for recognition of these species due to more intra- and interspecific sequence divergences of this gene. DNA barcodes and a novel species-specific multiplex PCR assay were used for accurate and rapid identification. Also, specific anti-venoms against these species were produced 
for protection of envenomed victims [53]. In the first cohort study, snake species identification based on the PCR technique was performed with a clinical approach in southern Nepal by nearly 749 snakebite patients with snake species in $25.9 \%$ cases being identified. Interestingly, the majority of envenomed patients were bitten by non-venomous snake's species [54]. Another method called peptide barcoding was introduced and tested for scorpions. In this approach, the extracted venom of scorpions through a simple protocol was used for identification of species by MALDI-TOF mass fingerprints database of venom barcoding [55].

\section{Antivenomics}

Selection of and treatment with specific anti-venoms constitute an important step in the treatment of envenomed victims. Choosing specific anti-venoms is a decision made by clinicians which depends on the specific species of venomous animals. Antivenoms are obtained from immunized animals; use nonspecific anti-venoms, due to inaccurate recognition of venomous animal species or inaccessibility of monospecific antivenom, triggers severe immune responses with various side effects even the probability of mortality in some envenomed victims [41].

Antivenomics is a proteomics-based method which provides immunological cross-reactivity profile of venom toxins which is exactly recognized by antibodies and toxins without or with poor recognition by the antivenom antibodies. To investigate the efficacy of antivenoms, we need information from both neutralization effects of antivenom in the body and antivenomics analysis data [56]. Based on advances of the antivenomics methodology, there are three generations of antivenomics methods. The first generation of antivenomics is an approach for identifying both immunoprecipitated antigens and free antigens of venom which are not bound to antibodies by RP-HPLC technique. The second generation first identifies the bound and unbound venom antigens and provides a quantitative comparison with the whole venom chromatogram. Finally, the quality and quantity of antivenom with a range of venom-antivenom concentrations are analyzed to determine a certain amount of antivenom for neutralization of a specific amount of the venom in the third generation [57].

For overcoming the deficiencies of the current antivenoms for treatment of envenomed victims, antivenomics creates the opportunity through we can investigate different anivenoms against various venoms. This method reveals the satisfactory function or insufficient neutralization of the current antivenoms. For some species for which there is no specific antivenom, hetero-specific and polyvalent antivenoms have been tested for immunological cross-reactivity by antivenomics analysis. The neutralization of important toxins with dangerous effects depends on the venom composition of species which determines whether the antivenom is appropriate for the treatment of envenomed victims [58] or not $[59,60]$. Although the gold standard for antivenom production has been their neutralization efficacy for venom lethality effects, according to the venom composition and its pathophysiologic effects, neutralization of other toxins with various effects by antivenoms should also be considered [61]. For example, the venom of Crotalus durissus subspecies in Brazil contains crotoxin which is the main toxin with neurotoxicity and myotoxicity effects plus crotamine with lower toxicity and induction of skeletal muscle spasms. Crotalic antivenom failed to recognize or neutralize crotamine; probably the venom injected to the animal for the antivenom production was devoid of crotamine. Alternatively, it could be attributed to the lower molecular weight of crotamine for stimulating the animal immune system. Therefore, we can apply the venomics and antivenomics analysis for optimizing antivenoms [62].

In addition to the investigation of the current antivenoms, there are reports of various insights into the production of novel antivenoms such as small-molecule inhibitors against components with enzymatic activity, aptamers based on peptide or oligonucleotide and recombinant antibodies against components with or without enzymatic activity. Thus, antivenom development depends on venom composition and complexity especially toxins and their function $[63,64]$.

\section{Conclusion}

Venom is the cause of mortality and morbidity of humans in the human and venomous animal interactions. In most cases, venom detection is based on the evidence presented by the envenomed patient or reports of a herpetologist. Therefore, there is an essential requirement for venom detection with more functional properties. In recent years, there have been many efforts for accurate detection and treatment with specific antivenoms based on molecular approaches. According to the advancements in the venom studies, there will be new opportunities in the treatment of envenomed victims and many other diseases in the future.

\section{Acknowledgement}

The authors would like to express gratitude for faculty members at Department of Medical Genetics, School of Advanced Technologies in Medicine, Golestan University of Medical Sciences.

\section{Author contributions}

$\mathrm{M} \mathrm{O}$ and $\mathrm{N} Y$ contributed to designing and composing of the review. S O, M M, and M O Prepared the first draft. F K-L and A A Critically revised the draft. All authors approved the final manuscript.

\section{References}

1. Casewell NR, Wüster W, Vonk FJ, Harrison RA, Fry BGJT, et al Complex cocktails: the evolutionary novelty of venoms. Trends Ecol Evol. 2013; 28: 219-229.

PubMed: https://pubmed.ncbi.nlm.nih.gov/23219381/ 
2. Jiang $Y$, Li Y, Lee $W, X u X$, Zhang $Y$, et al. Venom gland transcriptomes of two elapid snakes (Bungarus multicinctus and Naja atra) and evolution of toxin genes. BMC Genomics. 2011; 12 : 1. PubMed: https://pubmed.ncbi.nlm.nih.gov/21194499/

3. Kordiš D, Gubenšek FJG. Adaptive evolution of animal toxin multigene families. Gene. 2000; 261: 43-52.

PubMed: https://pubmed.ncbi.nlm.nih.gov/11164036/

4. Aird SD, Arora J, Barua A, Qu L, Terada K, et al. Population genomic analysis of a pitviper reveals microevolutionary forces underlying venom chemistry. Genome Biol Evol. 2017; 9: 2640-2649.

PubMed: https://pubmed.ncbi.nlm.nih.gov/29048530/

5. Zhang Y. Why do we study animal toxins? Dongwuxue Yanjiu. 2015 36: 183-122.

PubMed: https://pubmed.ncbi.nlm.nih.gov/26228472/

6. Brahma RK, McCleary RJ, Kini RM, Doley RJT. Venom gland transcriptomics for identifying, cataloging, and characterizing venom proteins in snakes. Toxicon. 2015; 93: 1-10.

PubMed: https://pubmed.ncbi.nlm.nih.gov/25448392/

7. Jahdasani R, Jamnani FR, Behdani M, Habibi-Anbouhi M, Yardehnavi $\mathrm{N}$, et al. Identification of the immunogenic epitopes of the whole venom component of the Hemiscorpius lepturus scorpion using the phage display peptide library. Toxicon. 2016; 124; 83-93.

PubMed: https://pubmed.ncbi.nlm.nih.gov/27845058/

8. Kazemi-Lomedasht F, Khalaj V, Bagheri KP, Behdani M, Shahbazzadeh D. The first report on transcriptome analysis of the venom gland of Iranian scorpion, Hemiscorpius lepturus. Toxicon. 2017; 125: 123-130. PubMed: https://pubmed.ncbi.nlm.nih.gov/27914888/

9. Klupczynska A, Pawlak M, Kokot Z, Matysiak JJT. Application of metabolomic tools for studying low molecular-weight fraction of animal venoms and poisons. Toxins (Basel). 2018; 10: 306.

PubMed: https://pubmed.ncbi.nlm.nih.gov/30042318/

10. Leonardi A, Biass D, Kordiš D, Stöcklin R, Favreau P, et al. Conus consors snail venom proteomics proposes functions, pathways, and novel families involved in its venomic system. J Proteome Res. 2012; 11: 5046-5058.

PubMed: https://pubmed.ncbi.nlm.nih.gov/22928724/

11. Park D, Jung JW, Choi BS, Jayakodi M, Lee J, et al. Uncovering the novel characteristics of Asian honey bee, Apis cerana, by whole genome sequencing. BMC Genomics. 2015; 16 : 1.

PubMed: https://pubmed.ncbi.nlm.nih.gov/25553907/

12. Torabi E, Asgari S, Khalaj V, Behdani M, Kazemi-Lomedasht F, et al Corrigendum to" The first report on transcriptome analysis of the venom gland of Iranian scorpion, Hemiscorpius lepturus Toxicon. 125 (2017) 123-130]. Toxicon. 2017; 128: 60.

PubMed: https://pubmed.ncbi.nlm.nih.gov/28192687/

13. Tasoulis T, Isbister GK. A review and database of snake venom proteomes. Toxins (Basel). 2017; 9: 290.

PubMed: https://pubmed.ncbi.nlm.nih.gov/28927001/

14. Abdel-Rahman MA, Omran MA, Abdel-Nabi IM, Ueda H, McVean AJ. Intraspecific variation in the Egyptian scorpion Scorpio maurus palmatus venom collected from different biotopes. Toxicon. 2009; 53: 349-359. PubMed: https://pubmed.ncbi.nlm.nih.gov/19103215/

15. Ruiming Z, Yibao M, Yawen H, Zhiyong D, Yingliang $W$, et al Comparative venom gland transcriptome analysis of the scorpion Lychas mucronatus reveals intraspecific toxic gene diversity and new venomous components. BMC Genomics. 2010; 11: 452. PubMed: https://pubmed.ncbi.nlm.nih.gov/20663230/

16. He Y, Zhao R, Di Z, Li Z, Xu X, et al. Molecular diversity of Chaerilidae venom peptides reveals the dynamic evolution of scorpion venom components fromButhidaetonon-Buthidae.JProteomics. 2013;89:1-14 PubMed: https://pubmed.ncbi.nlm.nih.gov/23774330/

17. Ma Y, He Y, Zhao R, Wu Y, Li W, et al. Extreme diversity of scorpion venom peptides and proteins revealed by transcriptomic analysis: implication for proteome evolution of scorpion venom arsenal. J Proteomics. 2012; 75: 1563-1576.

PubMed: https://pubmed.ncbi.nlm.nih.gov/22155128/

18. Ma J, Shi YB. The Mesobuthus martensii genome reveals the molecular diversity of scorpion toxins. Cell Biosci. 2014; 4: 1. PubMed: https://pubmed.ncbi.nlm.nih.gov/24383941/

19. Ma Y, Zhao Y, Zhao R, Zhang W, He Y, et al. Molecular diversity of toxic components from the scorpion Heterometrus petersii venom revealed by proteomic and transcriptome analysis. Proteomics. 2010; 10: 2471-2485.

PubMed: https://pubmed.ncbi.nlm.nih.gov/20443192/

20. Rendón-Anaya M, Delaye L, Possani LD, Herrera-Estrella AJ. Global transcriptome analysis of the scorpion Centruroides noxius: new toxin families and evolutionary insights from an ancestral scorpion species. PLoS One. 2012; 7: e43331.

PubMed: https://www.ncbi.nlm.nih.gov/pmc/articles/PMC3422302/

21. Romero-Gutiérrez M, Santibáñez-López C, Jiménez-Vargas J, Batista C, Ortiz E, et al. Transcriptomic and proteomic analyses reveal the diversity of venom components from the vaejovid scorpion Serradigitus gertschi. Toxins (Basel). 2018; 10: 359.

PubMed: https://pubmed.ncbi.nlm.nih.gov/30189638/

22. Xu X, Duan Z, Di Z, He Y, Li J, et al. Proteomic analysis of the venom from the scorpion Mesobuthus martensii. J Proteomics. 2014; 106: 162-180. PubMed: https://pubmed.ncbi.nlm.nih.gov/24780724/

23. Al-Asmari AK, Kunnathodi F, Al Saadon K, Idris MM. Elemental analysis of scorpion venoms. J Venom Res. 2016; 7: 16-20. PubMed: https://pubmed.ncbi.nlm.nih.gov/27826410/

24. Himaya S, Lewis RJI. Venomics-accelerated cone snail venom peptide discovery. Int J Mol Sci. 2018; 19: 788. PubMed: https://pubmed.ncbi. nlm.nih.gov/29522462/

25. Tayo LL, Lu B, Cruz LJ, Yates III JR. Proteomic analysis provides insights on venom processing in Conus textile. J Proteome Res. 2010; 9: 2292-2301.

PubMed: https://pubmed.ncbi.nlm.nih.gov/20334424/

26. Dutt M, Dutertre S, Jin AH, Lavergne V, Alewood PF, et al. Venomics reveals venom complexity of the piscivorous cone snail, Conus tulipa. Mar Drugs. 2019; 17: 71

PubMed: https://www.ncbi.nlm.nih.gov/pmc/articles/PMC6356538/

27. Jin AH, Dutertre S, Dutt M, Lavergne V, Jones A, et al. TranscriptomicProteomic Correlation in the Predation-Evoked Venom of the Cone Snail, Conus imperialis. Mar Drugs. 2019; 17: 177.

PubMed: https://www.ncbi.nlm.nih.gov/pmc/articles/PMC6471084/

28. Safavi-Hemami H, Hu H, Gorasia DG, Bandyopadhyay PK, Veith PD, et al. Combined proteomic and transcriptomic interrogation of the venom gland of Conus geographus uncovers novel components and functional compartmentalization. Mol Cell Proteomics. 2014; 13: 938-953. PubMed: https://pubmed.ncbi.nlm.nih.gov/24478445/

29. Phuong MA, Mahardika GN, Alfaro ME. Dietary breadth is positively correlated with venom complexity in cone snails. BMC Genomics. 2016; 17: 401.

PubMed: https://pubmed.ncbi.nlm.nih.gov/27229931/

30. Dutertre S, Jin AH, Alewood PF, Lewis RJ. Intraspecific variations in Conus geographus defence-evoked venom and estimation of the human lethal dose. Toxicon. 2014; 91: 135-144. PubMed: https://pubmed.ncbi.nlm.nih.gov/25301479/

31. Dutertre S, Jin AH, Vetter I, Hamilton B, Sunagar K, et al. Evolution of separate predation-and defence-evoked venoms in carnivorous cone snails. Nat Commun. 2014; 5: 3521.

PubMed: https://pubmed.ncbi.nlm.nih.gov/24662800/

32. Lomazi RL, Nishiduka ES, Silva PI, Tashima AK. Identification of Peptides in Spider Venom Using Mass Spectrometry Peptidomics. methods mol boil. 2018; 359-367.

PubMed: https://pubmed.ncbi.nlm.nih.gov/29476524/ 
33. Diniz MR, Paiva AL, Guerra-Duarte C, Nishiyama Jr, MY, Mudadu MA et al. An overview of Phoneutria nigriventer spider venom using combined transcriptomic and proteomic approaches. PLoS One. 2018; 13: e0200628.

PubMed: https://pubmed.ncbi.nlm.nih.gov/30067761/

34. He Q, Duan Z, Yu Y, Liu Z, Liu Z, et al. The venom gland transcriptome of Latrodectus tredecimguttatus revealed by deep sequencing and cDNA library analysis. PLoS One. 2013; 8: e81357.

PubMed: https://pubmed.ncbi.nlm.nih.gov/24312294/

35. Oldrati V, Koua D, Allard PM, Hulo N, Arrell M, et al. Peptidomic and transcriptomic profiling of four distinct spider venoms. PLoS One. 2017; 12: e0172966.

PubMed: https://pubmed.ncbi.nlm.nih.gov/28306751/

36. Santana R, Perez D, Dobson J, Panagides N, Raven R, et al. Venom profiling of a population of the theraphosid spider phlogius crassipes reveals continuous ontogenetic changes from juveniles through adulthood. Toxins (Basel). 2017; 9: 116.

PubMed: https://pubmed.ncbi.nlm.nih.gov/28346332/

37. Zancolli G, Sanz L, Calvete J, Wüster W. Venom on-a-chip: a fast and efficient method for comparative venomics. Toxins (Basel). 2017; 9: 179. PubMed: https://pubmed.ncbi.nlm.nih.gov/28555029/

38. Carregari VC, Rosa-Fernandes L, Baldasso P, Bydlowski SP, Marangoni S, et al. Snake Venom Extracellular vesicles (SVEVs) reveal wide molecular and functional proteome diversity. Sci Rep. 2018; 8: 12067.

PubMed: https://pubmed.ncbi.nlm.nih.gov/30104604/

39. de Oliveira UC, Nishiyama Jr, MY, dos Santos MBV, de Paula Santosda-Silva $\mathrm{A}$, de Menezes Chalkidis $\mathrm{H}$, et al. Proteomic endorsed transcriptomic profiles of venom glands from Tityus obscurus and $T$. serrulatus scorpions. PLoS One. 2018; 13: e0193739.

PubMed: https://pubmed.ncbi.nlm.nih.gov/29561852/

40. Ranawaka UK, Lalloo DG, de Silva HJ. Neurotoxicity in snakebite-the limits of our knowledge. PLoS Negl Trop Dis. 2013; 7: e2302. PubMed: https://pubmed.ncbi.nlm.nih.gov/24130909/

41. Theakston RGD, Laing GD. Diagnosis of snakebite and the importance of immunological tests in venom research. 2014; 6: 1667-1695. PubMed: https://pubmed.ncbi.nlm.nih.gov/24859244/

42. Suntrarachun S, Pakmanee N, Tirawatnapong T, Chanhome L, Sitprija VJT. Development of a polymerase chain reaction to distinguish monocellate cobra (Naja khouthia) bites from other common Thai snake species, using both venom extracts and bite-site swabs. Toxicon. 2001; 39: 1087-1090.

PubMed: https://pubmed.ncbi.nlm.nih.gov/11223099/

43. Selvanayagam ZE, Gopalakrishnakone P. Tests for detection of snake venoms, toxins and venom antibodies: review on recent trends (19871997). Toxicon. 1999; 37: 565-586.

PubMed: https://pubmed.ncbi.nlm.nih.gov/10082159/

44. Minton SA. Present tests for detection of snake venom: clinical applications. Ann Emerg Med. 1987; 16: 932-937.

PubMed: https://pubmed.ncbi.nlm.nih.gov/3307554/

45. Chandler HM, Hurrell JG. A new enzyme immunoassay system suitable for field use and its application in a snake venom detection kit. Clin Chim Acta. 1982; 121: 225-230.

PubMed: https://pubmed.ncbi.nlm.nih.gov/7046996/

46. Trevett A, Lalloo D, Nwokolo N, Theakston R, Naraqi S, et al. Venom detection kits in the management of snakebite in Central province, Papua New Guinea. Toxicon. 1995; 33: 703-705.

PubMed: https://pubmed.ncbi.nlm.nih.gov/7660375/

47. Eng KH, Gopalakrishnakone PJ. Immunogenicity of venoms from four common snakes in the South of Vietnam and development of ELISA kit for venom detection. J Immunol Methods. 2003; 282: 13-31.

PubMed: https://pubmed.ncbi.nlm.nih.gov/14604537/

48. Ibrahim NM, Saleh NS, Aati AMA, El-kady EMJ. Detection of venoms from Egyptian elapids in experimentally envenomed mice. 2014; 6.
49. Eng KH, Gopalakrishnakone PJ. Bioelectronics. Optical immunoassay for snake venom detection. 2004; 19: 1285-1294. Biosens Bioelectron. PubMed: https://pubmed.ncbi.nlm.nih.gov/15046761/

50. Choudhury SN, Konwar B, Kaur S, Doley R, Mondal BJ. Study on snake venom protein-antibody interaction by surface plasmon resonance spectroscopy. 2018; 8: 193-202.

51. Teja GKA, More N, Kapusetti GJ, Medicine H. Advanced Biosensorbased Strategy for Specific and Rapid Detection of Snake Venom for Better Treatment. 2018; 3: 61-67.

52. Pook $\mathrm{C}$, McEwing R. Mitochondrial DNA sequences from dried snake venom: a DNA barcoding approach to the identification of venom samples. Toxicon. 2005; 46: 711-715.

PubMed: https://pubmed.ncbi.nlm.nih.gov/16157361/

53. Supikamolseni A, Ngaoburanawit N, Sumontha M, Chanhome L, Suntrarachun S, et al. Molecular barcoding of venomous snakes and species-specific multiplex PCR assay to identify snake groups for which antivenom is available in Thailand. Genet Mol Res. 2015; 14 : 13981-13997.

PubMed: https://pubmed.ncbi.nlm.nih.gov/26535713/

54. Sharma SK, Kuch U, Höde P, Bruhse L, Pandey DP, et al. Use of molecular diagnostic tools for the identification of species responsible for snakebite in Nepal: a pilot study. PLoS Negl Trop Dis. 2016; 10: e0004620.

PubMed: https://pubmed.ncbi.nlm.nih.gov/27105074/

55. Schaffrath S, Predel RJ. A simple protocol for venom peptide barcoding in scorpions. 2014; 3: 239-245.

56. Calvete JJ, Gutiérrez JM, Sanz L, Pla D, Lomonte BJ. Antivenomics: A Proteomics Tool for Studying the Immunoreactivity of Antivenoms. 2015.

57. Ledsgaard L, Jenkins T, Davidsen K, Krause K, Martos-Esteban A, et al Antibody Cross-Reactivity in Antivenom Research. Toxins (Basel). 2018; 10: 393.

PubMed: https://pubmed.ncbi.nlm.nih.gov/30261694/

58. Tan CH, Tan KY, Ng TS, Quah ES, Ismail AK, et al. Venomics of Trimeresurus (Popeia) nebularis, the Cameron Highlands pit viper from Malaysia: Insights into venom proteome, toxicity and neutralization of antivenom. Toxins (Basel). 2019; 11: 95.

PubMed: https://pubmed.ncbi.nlm.nih.gov/30736335/

59. Patra A, Kalita B, Chanda A, Mukherjee AK. Proteomics and antivenomics of Echis carinatus carinatus venom: Correlation with pharmacological properties and pathophysiology of envenomation. Sci Rep. 2017; 7: 17119.

PubMed: https://pubmed.ncbi.nIm.nih.gov/29215036/

60. Tan $\mathrm{KY}$, Tan $\mathrm{NH}$, Tan $\mathrm{CH}$. Venom proteomics and antivenom neutralization for the Chinese eastern Russell's viper, Daboia siamensis from Guangxi and Taiwan. Sci Rep. 2018; 8: 8545.

PubMed:https://pubmed.ncbi.nlm.nih.gov/29867131/

61. Gutiérrez J, Solano G, Pla D, Herrera M, Segura Á, et al. Preclinical evaluation of the efficacy of antivenoms for snakebite envenoming: state-of-the-art and challenges ahead. Toxins (Basel). 2017; 9: 163. PubMed: https://pubmed.ncbi.nlm.nih.gov/28505100/

62. Teixeira-Araújo R, Castanheira P, Brazil-Más L, Pontes F, de Araújo ML, et al. Antivenomics as a tool to improve the neutralizing capacity of the crotalic antivenom: a study with crotamine. J Venom Anim Toxins Incl Trop Dis. 2017; 23: 28.

PubMed: https://pubmed.ncbi.nlm.nih.gov/28507562/

63. Knudsen C, Laustsen AJT. Recent advances in next generation snakebite antivenoms. Trop Med Infect Dis. 2018; 3: 42. PubMed: https://pubmed.ncbi.nlm.nih.gov/30274438/

64. Laustsen AH. Guiding recombinant antivenom development by omics technologies. N Biotechnol. 2018; 45: 19-27.

PubMed: https://pubmed.ncbi.nlm.nih.gov/28552814/ 